\title{
Deflection Routing in OBS Networks
}

\author{
M.Thachayani \\ Department of Electronics and Communication \\ Engineering, \\ Pondicherry Engineering College, \\ Puducherry-605014, India
}

\author{
R.Nakkeeran, \\ Department of Electronics, \\ School of Engineering, \\ Pondicherry University, \\ Puducherry-605014, India
}

\begin{abstract}
Optical Burst Switching (OBS) network proposed as future optical internet is capable of carrying bursty traffic and adapting to the present technological constraints. Since the OBS network is buffer-less in nature, contention resolution using deflection technique received much attention. Deflection routing can work with limited optical buffering or even no buffering. This paper gives an overview of the research done so far in this area of deflection routing in OBS networks.
\end{abstract}

Keywords: Optical Burst Switching, contention resolution, deflection routing, alternate path selection, congestion

\section{INTRODUCTION}

Optical Burst Switching (OBS) network is emerging as a promising candidate for future optical internet due to its ability to carry bursty traffic, adapting to the technological constraints in All Optical Networks (AON). OBS concept has been introduced in the year 1999 [1] and till date lot of researches are going on in this area to make this network a reality.

In OBS networks, the edge nodes aggregate the incoming traffic into variable length optical bursts and core nodes asynchronously switch these bursts. Figure 1 illustrates the basic concept of the OBS network. The key characteristic of OBS is the hybrid approach in which burst control packets are signaled out of band and processed electronically while data bursts stay in the optical domain until they reach their destination node. This avoids buffering as well as synchronization problem that are present in optical packet switching. The one-pass reservation ensures elimination of significant signaling delay. However, due to this one-pass reservation strategy and statistical multiplexing, burst loss can occur in case of contention and efficient resolution strategies in OBS core nodes are essential in order to achieve a low burst blocking probability.

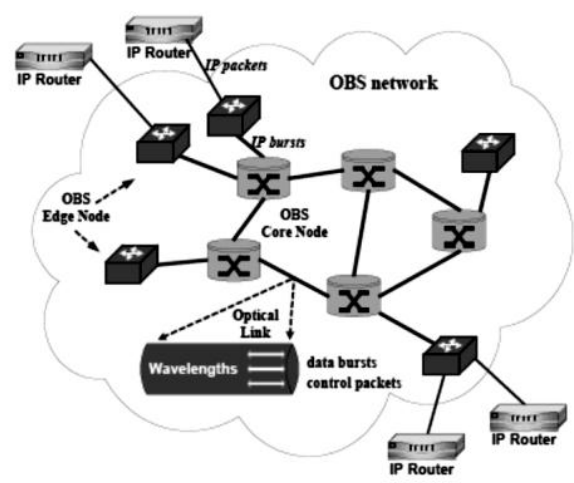

Figure 1 Schematic diagram of OBS network
Though numerous approaches like optical buffering, and wavelength conversion had been proposed to solve the wavelength contention problem in OBS, due to the technological and economical limitations most of the schemes seem to be impractical [2], [3] and [4].

Deflection routing can work with limited optical buffering or even without buffering because the contending bursts are deflected to an available output port rather than delaying them. The basic idea of the deflection routing is to utilize other unused links in the network. Since different output links are selected for the contending bursts in a deflection routed network, contention can be avoided. However it also has its limitations and problems. This paper reviews the research so far carried out in deflection routed OBS networks.

\section{DEFLECTION IN OBS NETWORKS}

Prior to the emergence of OBS networks, deflection routing was first used as a contention resolution method in optical networks with regular mesh topologies [5].

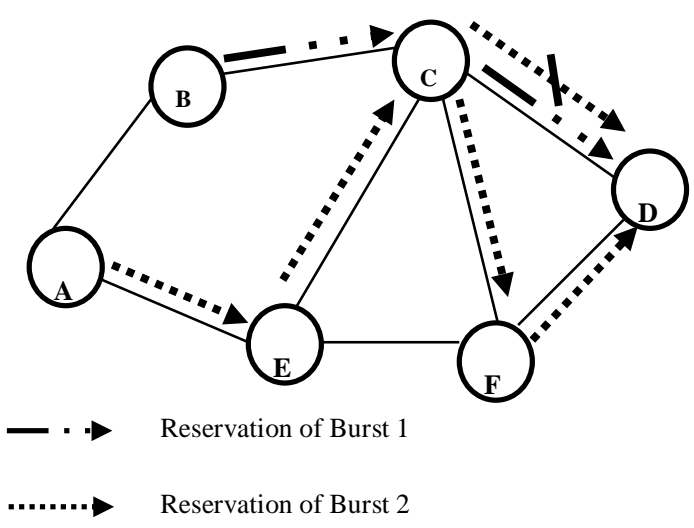

Figure 2 Conceptual view of deflection routing

It was observed that the performance of deflection routing is better than the hot-potato routing in a network with highconnectivity topology, such as Shuffle Net [6], [7]. Routing heuristics were proposed to enhance the performance of deflection routing [8]. 


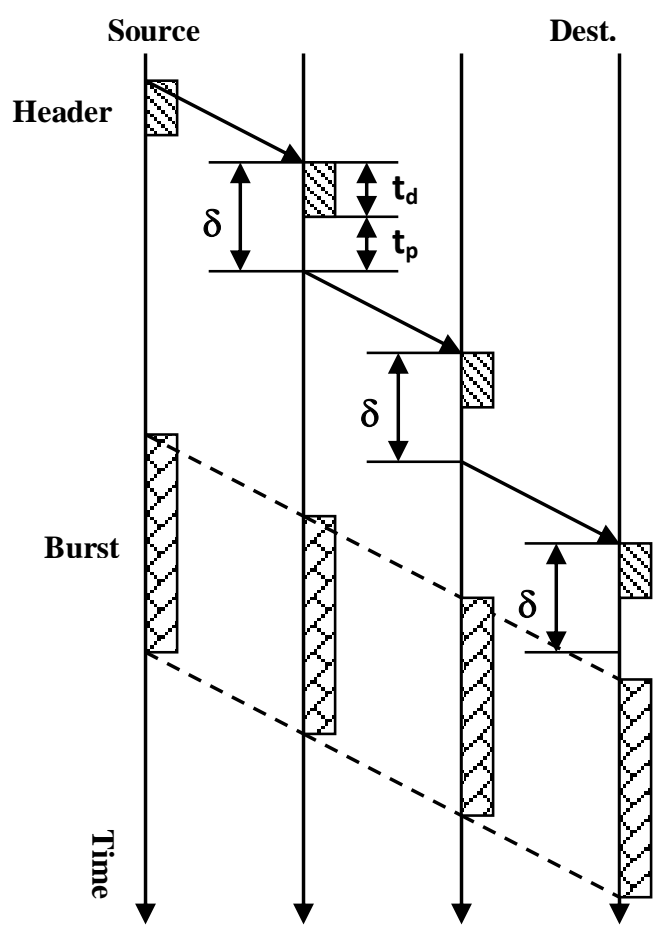

3(a)

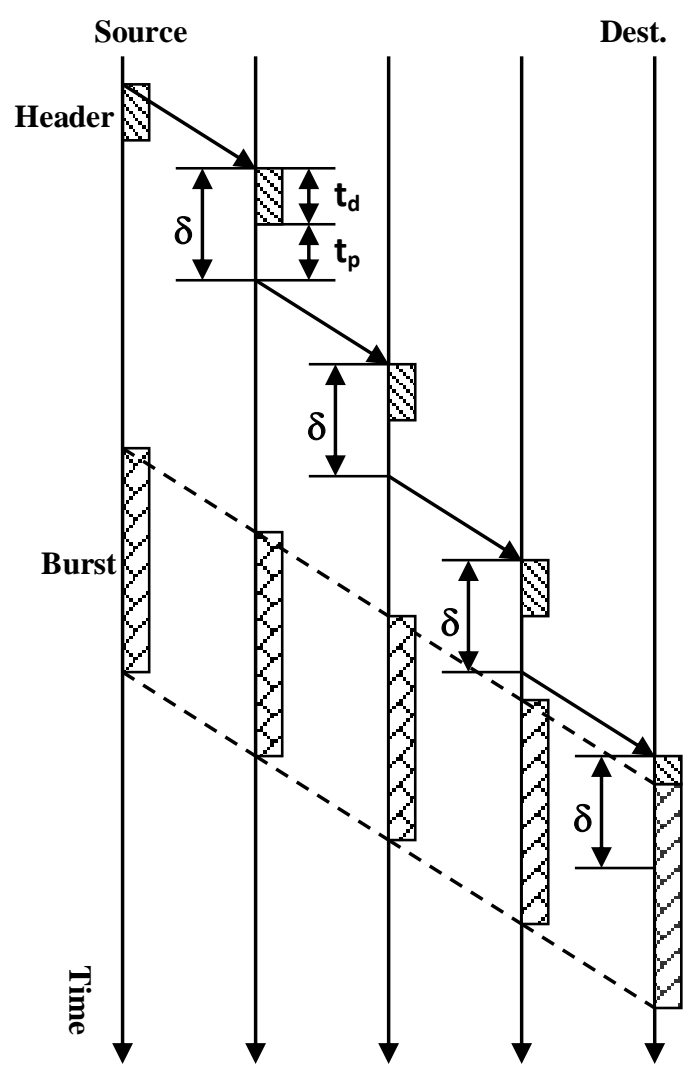

3(b)

Figure 3 Timeline of burst and header, 3(a) Without deflection, 3(b) With deflection

With the emergence of OBS technology, a deflection routing protocol for OBS network was proposed [9] and variants of the basic protocol were developed. The process of deflection in OBS can be explained as follows.

Consider the situation where a burst transmission request arrives at a given node and cannot be accommodated on any output fiber that could connect the burst to its designated next hop. Then the burst may be deflected from its original next hop onto a fiber connected to a different next hop node that can accommodate the burst. It then becomes the responsibility of the alternate next hop node to re-route the burst on to its destination. Sometimes it is possible that this deflected route may even pass back through the node at which the burst was originally deflected.

Figure 2 gives a clear view of this deflection routing concept. Let the bursts 1 and 2 generated by nodes $\mathrm{B}$ and $\mathrm{A}$ respectively are destined to the destination $\mathrm{D}$ and the routes selected are B-C-D and A-E-C-D. Assume that there is no wavelength conversion and both the bursts are using the same wavelength. At node $\mathrm{C}$, the reservation request for burst 2 cannot be accepted due to contention. If deflection is used this can be rerouted via node F (i.e., A-E-C-F-D instead of A-E-CD).

Early arrival or the insufficient offset problem is an important issue with deflection routed OBS networks. It can be explained as follows (refer Figure 3). In OBS networks the data burst follows the control packet after a time delay called "the offset time". Let the original offset time at the source (edge router) be $\boldsymbol{T}$ time units. If a control packet has to encounter $\boldsymbol{H}$ nodes along the burst's path through the network, then it will be delayed by processing at each of the $\boldsymbol{H}$ hops. The residual offset time at hop $j$ is the remaining offset between the arrival of the control packet at hop $j$ and the arrival of the burst at hop $j$. Referring to the timelines for the burst and control packet shown in Figure 3, we can determine the conditions required for the offset time. The control packet is transmitted at the edge router, with a transmission delay of $\boldsymbol{t}_{\boldsymbol{d}}$ seconds. After being read at node 1 on the output of link 1, it is processed by the ECU (Electronic Control Unit) and incurs a processing delay of $t_{p}$. It is then transmitted on link 2, incurring a processing delay of another $t_{p}$ seconds. The process then repeats at all intermediate nodes. At the destination, we assume that the control packet requires a final processing delay. Since both the burst and control packet are propagating with the same delay, propagation delays do not affect the residual offset times.

From figure 3(a), we can deduce that the residual offset time at the destination, must be greater than the final transmission delay and processing delay, to avoid a burst arriving at the destination ahead of its control packet. In other words, the initial offset time $\boldsymbol{T} \geq\left(\boldsymbol{t}_{p}+\boldsymbol{t}_{d}\right) \boldsymbol{H}$,

$$
\begin{array}{r}
\text { where } \boldsymbol{t}_{\boldsymbol{d}} \text {-transmission delay per hop, } \\
\boldsymbol{t}_{\boldsymbol{p}} \text { - processing delay per node, } \\
\boldsymbol{H} \text {-number of hops in the path }
\end{array}
$$

If the offset time is insufficient, the burst may arrive at a node before its control packet and this is termed as an early arrival. In network with deflection routing, generally the deflected burst follows a longer path than the primary route and this excess delay should be accounted for to avoid the early arrival. This is illustrated in Figure 3 (b). In this case, due to deflection, the burst undergoes an additional hop and this is not accounted in initial offset time. It can be seen that the 
burst arrives before the entire control packet is received by the destination and hence it will be lost.

In the basic deflection method used idle optical links are used as fiber delay lines for contention resolution. A burst may be deflected back to the sender again and this may result in shortterm loops. However, a Time To Live (TTL) field is added in the header to prevent infinite loops. This scheme exhibits reduced burst loss and the average delay as compared with data retransmission from the source [9].

\section{DEFLECTION AND BUFFERS}

Deflection routing may be implemented with or without output buffers.

\subsection{Bufferless}

In bufferless deflection routing, the additional offset time required due to deflection must be added to the initial offset time at the source node. The number of times a burst gets deflected must be restricted to avoid the early arrival problem. The control packet contains the number of deflections and if this number is over the threshold value, the rerouted bursts are just dropped. An additional routing offset delay of $10 \%$ results in more than $50 \%$ reduction in contention [10].

Assigning appropriate offset delay is important since insufficient delay results in early arrival of bursts and larger delays will results in longer transmission delay. At very low loads, bursts may not be deflected and hence smaller offset delays are sufficient. Longer offset times are useful if the network is moderately loaded. Appropriate offset time may be dynamically assigned according to the load condition of the network. To implement dynamic delay, blocking probability need to be calculated at regular intervals based on the negative acknowledgements received at the source node. Based on this information, offset time may be determined using reinforcement learning. Dynamic offset time provides significant performance improvement over classical deflection routing [9]. However, the delay increases and in the worst case can be as high as 52 times [11].

When the number of times a burst gets deflected increases, the offset delay required will also increase. In bufferless networks, the initial offset delay must be large enough accounting for multiple deflections. But the entire delay may not be used often. Wavelength reservation approach is used to reduce the probability of repeated deflection [12]. In this scheme a particular number of wavelengths at every node are exclusively reserved for deflected bursts in every outgoing link. The wavelength reservation scheme marginally outperforms the limited buffers deflection scheme [13] in terms of overall blocking probability.

\subsection{Buffered}

Although deflection routing can be performed without buffers, the performance can be improved by including limited buffer. Two possible output buffered architectures namely share-perport and share-per-node are considered for OBS switch [13]. With the increase of either the length or the number of FDLs (Fiber Delay Lines), the blocking probability decreases. However, the performance gain reaches a limit (upper bound), when the network capacity almost saturates.

\section{HYBRID DEFLECTION SCHEMES}

Deflection routing is combined with other contention resolution schemes such as wavelength conversion. The performance achieved by combining the deflection routing and wavelength conversion is better than the individual schemes. Increasing the wavelength conversion range or increasing the level of deflection significantly reduces the mean burst blocking probability, particularly for low loads. Considering the individual schemes, deflection routing marginally outperforms the limited wavelength conversion [14]. The deflection routing can also be combined with retransmission scheme in such a way that the combined scheme always results in lesser end-to-end delay and burst loss ratio compared to PDR (Pure deflection routing) and PBR (Pure blocking and retransmission) [15]. The HDR (Hybrid deflection and retransmission) scheme transmits the data bursts first using deflection routing and if the deflection routing fails, applies burst retransmission.

The HDR performs better than both PDR and PBR up to certain load. At very high loads, there is an increased probability of a burst getting repeated deflections and retransmissions in case of HDR. Hence, the performance degrades and become worse than pure deflection and pure retransmission. To avoid this degradation, a hop count based constraint is used for limiting deflection. This is termed as LHDR (Limited hybrid deflection and retransmission). This limitation is found to improve the blocking as well as delay performance at high loads.

\section{LIMITED DEFLECTION METHODS}

It is well known that the performance of deflection routing will degrade when the traffic load is beyond some threshold for an unslotted system [5], [9] and [16]. This is applicable to OBS networks as well and hence the deflection should be limited during heavy load condition to prevent instability of the network. Providing limited FDLs or access control of the local traffic was suggested in order to keep the network stable [5], [16].

This limitation on deflection may be introduced using different approaches. One such approach is to deflect a burst with a particular probability instead of deflecting always, when contention occurs [17]. The value of this deflection probability can be set before operation according to statistical records or adjusted dynamically based on the traffic load.

Another approach to limit deflection is to reserve a particular number of wavelengths on each link exclusively for primary bursts [18]. This wavelength reservation scheme alleviates the destabilizing effect and increases the throughput at high loads. Preemptive priority is a similar method in which a first-choice burst is given the right to preempt a reservation that has been scheduled for a deflected burst [19]. Preemptive priority has a negligible effect on blocking during stable periods and guarantees protection against destabilization during overloads.

However, it must be noted that at low loads, unprotected deflection routing may yield better performance than all the above mentioned protected deflection routing schemes.

Access or flow control approach may also be used to improve the performance of deflection routed OBS network under high loads. Leaky Bucket Deflection method, which has been used in Asynchronous Transfer Mode (ATM) can also be used in OBS (LB Deflection method) [20]. In this approach the transmission rate is restricted to a maximum value by means of generating tokens at a fixed rate. Data burst should acquire a token in order to get transmitted. This method absorbs the fluctuations of the network load and controls the burst loss 
probability even at high loads. Average delay is more in this method since it is influenced not only by the transmission delay but also by the admission delay.

\section{ALTERNATE PATH SELECTION BASED ON PERFORMANCE CONSTRAINTS}

The basic deflection routing simply routes the traffic to any alternate path available. The alternate path may also be selected based on certain performance metric. In general, performance metric is chosen such that congestion is avoided or minimized. A path may be selected based on minimization of a performance measure that combines distance and blocking due to contention. In such schemes optimization of alternate routes is carried out based on updates received from other nodes. Traffic considered may be of adjacent nodes or of the entire network. Since load balancing is achieved and heavily congested routes are avoided, these schemes perform better in terms of blocking performance. However, the additional delay and overhead is unavoidable [21], [22], [23]. The blocking performance can be further improved by introducing a $\mathrm{U}$ - turn option, which allows the bursts to come back to the original node if paths to all other nodes are unavailable [22].

Another approach is to use explicit congestion notification. The principle of these schemes is, when a node detects the congested link, it sends the congestion information to preceding nodes so that the data bursts can avoid this link [24]. In order to sense the traffic condition accurately, the moving average of some past ' $M$ ' usage rates may be used [25]. Whether the burst follows the shortest path or alternate non-shortest path is decided based on the congestion status of the neighboring nodes.

\section{INTEROPERABILITY WITH OTHER PROTOCOLS}

The behavior of TCP connections in optical burst switching networks with deflection routing is analyzed [26]. Deflection routing is found to provide improved performance. The aggregation of more packets out of one TCP flow in a burst has positive impact on TCP performance with deflection routing.

Dynamic deflection routing in a three-node OBS test bed is demonstrated experimentally. This confirmed the usefulness and viability of deflection routing in resolving contention and the possibility of high-speed Ether frame encapsulation in OBS [27].

\section{CONCLUSION}

In this paper an overview of the deflection routed OBS networks is presented. Deflection routing is a simple yet powerful contention resolution technique. It is particularly useful for OBS networks since it is not possible to have unlimited number of wavelength converters or buffers in OBS networks.

\section{REFERENCES}

[1] Qiao.C, Yoo.M, Optical burst switching (OBS) - a new paradigm for an optical Internet, Journal of High Speed Networks, Special issue on optical communications, vol. 8, no.1, pp. 69-84, 1999.
[2] C. M. Gauger, Martin Kohn, Joachim Scharf, Comparison of Contention Resolution Strategies in OBS Network Scenarios, ICTON 2004, vol. 1, pp.18-21, July 4-8, 2004.

[3] M. Yoo, C. Qiao, and S. Dixit, A comparative study of contention resolution policies in optical burst switched WDM networks, Proc. SPIE Int. Conf. Terabit Optical Networking, vol. 4213, pp. 124-135, Nov. 2000.

[4] C. Gauger, Performance of converter pools for contention resolution in optical burst switching, in Proc. SPIE Optical Netw. Commun. Conf. (OptiComm 2002), Boston, MA, pp. 109-117, July 2002.

[5] F. Borgonovo, L. Fratta, and J. A. Bannister, On the design of optical deflection-routing networks, in Proc. IEEE INFOCOM, vol. 1, pp. 120-129, Mar. 1994.

[6] F. Forghieri, A. Bononi, and P. R. Prucnal, Analysis and comparison of hot-potato and single-buffer deflection routing in very high bit rate optical mesh networks, IEEE T.O Communication, vol. 43, no. 1, pp. 88-98, Jan. 1995.

[7] A. Bononi, G. A. Castanon, and O. K. Tonguz, Analysis of hot-potato optical networks with wavelength conversion, IEEE Journal of Lightwave Technology, vol. 17, no. 4, pp. 525-534, Apr. 1999.

[8] T. Chich, J. Cohen, and P. Fraigniaud, Unslotted deflection routing: A practical and efficient protocol for multi-hop optical networks, IEEE/ACM Trans. on Networks, vol. 9, pp. 47-59, Feb. 2001.

[9] X. Wang, H. Morikawa, and T. Aoyama, Burst optical deflection routing protocol for wavelength routing WDM networks, in Proc. IEEE OptiComm, pp. 257-266, 2000.

[10] S. Kim, N. Kim, and M. Kang, Contention resolution for optical burst switching networks using alternate routing, Proc. IEEE ICC'02, vol. 5, pp. 2678-2681, April 2002.

[11] A. Belbekkouche and A Hafid, An adaptive reinforcement learning-based approach to reduce blocking probability in buffer-less OBS networks, Proc. ICC'07,

pp. 2377-2382, 2007.

[12] Danka Pevac and Miroslav Pevac, The influence of a wavelength allocation scheme to an Optical Burst Switching node performance, Proc. EUROCON'07, pp.1068 - 1072, Sept. 9-12, 2007.

[13] C. F. Hsu, T. L. Liu, and N. F. Huang, Performance analysis of deflection routing in optical burst switched networks, Proc. INFOCOM'02, vol. 1, pp. 55-73 June 2002.

[14] A. Zalesky, H. L. Vu, Z. Rosberg, E. Wong, and M. Zukerman, Evaluation of Limited Wavelength Conversion and Deflection Routing as Methods to Reduce Blocking Probability in Optical Burst Switched Networks, Proc. ICC’04, vol. 3, June 2004.

[15] Son-Hong Ngo, Xiaohong Jiang and Susumu Horiguchi, Hybrid Deflection and Retransmission Routing Schemes for OBS Networks, IEEE Workshop on High Performance switching and routing, June- 2006, Digital Object Identifier 10.1109/HPSR.2006.1709739 
[16] F. Borgonovo, L. Fratta, and J.A. Bannister, Unslotted deflection routing in all-optical networks, in Proc. of GLOBECOM, vol. 1, pp. 119-125, 1993.

[17] Y. Chen, H. Wu, D. Xu, and C. Qiao, Performance analysis of optical burst switched node with deflection routing, Proc. ICC'03, vol. 2, pp. 1355-1359, May 2003.

[18] A. Zalesky, H. L. Vu, Z. Rosberg, E. Wong, and M. Zukerman, Modeling and performance evaluation of optical burst switched networks with deflection routing and wavelength reservation, in Proc. IEEE INFOCOM, vol. 3, pp. 1864-1871, March 2004.

[19] A. Zalesky, H.L. Vu, Z. Rosberg, E. Wong and M. Zukerman, Stabilizing Deflection Routing in Optical Burst Switched Networks, IEEE Journal On Selected Areas In Communications, vol. 25, Issue 06, pp. 3-19, August 2007.

[20] tYoshihiko MORI, et al, Effective Flow-rate Control for the Deflection Routing based Optical Burst Switching Networks, Proc. Asia - Pacific Conf. on Communication, APCC'06, pp. 1-5, August 2006.

[21] S. Lee, K. Sriram, H. Kim, and J. Song, ContentionBased Limited Deflection Routing Protocol in Optical Burst-Switched Networks, IEEE Journal On Selected Areas In Communications, vol. 23, no. 8, pp. 15961611, Aug. 2005.

[22] T. Coutelen, H. Elbiaze, B. Jaumard and A. Metnani, Measurement-Based Alternative Routing Strategies in
Optical Burst-Switched Networks, Proc. ICTON 2005, pp. $224-227$, vol. 1, 3-7 July 2005.

[23] Y. Du, C. Zhu, X. Zheng, Y. Guo, H. Zhang, A Novel Load Balancing Deflection Routing Strategy in Optical Burst Switching Networks, National Fiber Optic Engineers Conference, NFOEC '07, pp. 1 - 3, 25-29 March 2007.

[24] Hiroki, TANIDA, Katsutoshi OHMAE, Young-Bok Choi, Hiromi OKADA, An Effective BECN /CRN Typed Deflection Routing for QoS Guaranteed Optical Burst Switching, IEEE GLOBECOM '03, no. 1, pp. 2601-2606, Dec. 2003.

[25] Hongtao PAN, Tomohiro ABE, Yoshihiko MORI, Young-Bok Choi, Hiromi OKADA, Feedback-based Load Balancing Routing for Optical Burst Switching Networks, Proc. Asia-Pacific Conf. on Communications, pp. 1033 - 1037, Oct. 2005.

[26] Michael Schlosser, Erwin Patzak, Philipp Gelpke, Impact of Deflection Routing on TCP Performance in Optical Burst Switching Networks, ICTON 2005, vol. 1, pp. 220-223, 3-7 July 2005.

[27] Abdullah Al Amin, Mitsuru Takenaka, et,al., Demonstration of Deflection Routing With Layer-2 Evaluation at $40 \mathrm{~Gb} / \mathrm{s}$ in a Three-Node Optical Burst Switching Testbed, IEEE Photonics Technology Letters, vol. 20, no. 3, pp. $178-180$, Feb. 2008. 